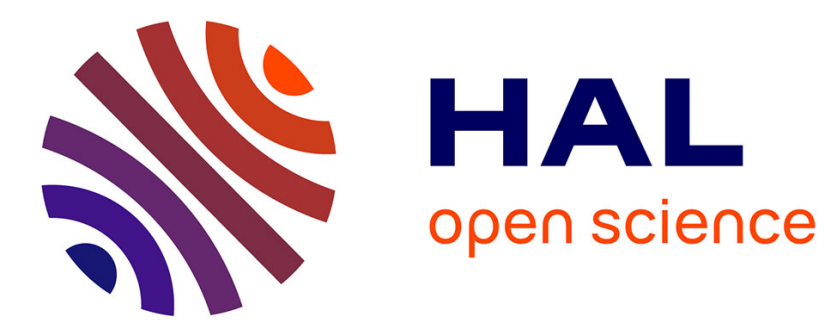

\title{
Elastocapillary adhesion of a soft cap on a rigid sphere
}

\author{
M Saint, H. Bense, M. Tani, M. Saint-Jean, E. Reyssat, B. Roman, J. Bico
}

\section{To cite this version:}

M Saint, H. Bense, M. Tani, M. Saint-Jean, E. Reyssat, et al.. Elastocapillary adhesion of a soft cap on a rigid sphere. Soft Matter, 2020, 16 (8), pp.1961-1966. 10.1039/c9sm02057h . hal-03015734

\section{HAL Id: hal-03015734 \\ https://hal.science/hal-03015734}

Submitted on 20 Nov 2020

HAL is a multi-disciplinary open access archive for the deposit and dissemination of scientific research documents, whether they are published or not. The documents may come from teaching and research institutions in France or abroad, or from public or private research centers.
L'archive ouverte pluridisciplinaire HAL, est destinée au dépôt et à la diffusion de documents scientifiques de niveau recherche, publiés ou non, émanant des établissements d'enseignement et de recherche français ou étrangers, des laboratoires publics ou privés. 


\title{
Elastocapillary adhesion of a soft cap on a rigid sphere
}

H.Bense, ${ }^{1,2}$ M.Tani, ${ }^{1,3}$ M.Saint Jean, ${ }^{1}$ E. Reyssat, ${ }^{1}$ B.Roman, ${ }^{1}$ and J.Bico ${ }^{1}$

1) Laboratoire PMMH, ESPCI Paris-PSL, CNRS UMR 7636, Sorbonne Université, Université de Paris, Paris,

France.

2) AMOLF, Science Park 104, 1098 XG Amsterdam, the Netherlands

${ }^{3)}$ Department of Physics, Tokyo Metropolitan University, Japan.

(Dated: 9 January 2020)

\begin{abstract}
We study the capillary adhesion of a spherical elastic cap on a rigid sphere of a different radius. Caps of small area accommodate the combination of flexural and in-plane strains induced by the mismatch in curvature, and fully adhere to the sphere. Conversely, wider caps delaminate and exhibit only partial contact. We determine the maximum size of the cap enabling full adhesion and describe its dependence on experimental parameters through a balance of stretching and adhesion energies. Beyond the maximum size, complex adhesion patterns such as blisters, bubbles or star shapes are observed. We rationalize these different states in configuration diagrams where stretching, bending and adhesion energies are compared through two dimensionless parameters.
\end{abstract}

\section{INTRODUCTION}

Wrapping a thin sheet on an adhesive sphere has been shown to generate a rich family of branched or oscillating patterns of adhesion ${ }^{1,2}$. More generally, crushing a sheet of paper in the hand leads to the development of crumpling singularities ${ }^{3}$. Such complexity is a consequence of the relatively high cost in stretching elastic energy involved in non-isometric deformations of thin sheets (proportional to the thickness $h$ of the sheet) in comparison with isometric bending (proportional to $h^{3}$ ). Indeed, following Gauss' seminal Theorema Egregium, mapping a plane into a sphere is a non-isometric transformation, and therefore generates tensile or compressive stresses along the surface ${ }^{4}$. Compressive stresses tend to induce wrinkles as observed in the mechanical embossing of a plate on a curved surface ${ }^{5-7}$ or when a flat thin sheet is deposited on the surface of a spherical droplet of water ${ }^{8}$. Conversely, regions under biaxial tension usually remain smooth and match the imposed geometry ${ }^{7}$. In the case of the wrapping of a stiff adhesive sphere by a naturally flat thin sheet, the typical width of the contact pattern is mainly dictated by a balance of stretching and adhesion energies, while bending energy is also involved in the selection of the adhesion pattern. We propose to extend this wrapping problem to the adhesion of a soft cap on a sphere of different curvature, a situation that could seem mundane for patients wearing contact lenses. Commercial contact lenses are indeed only available within a few discrete radii of curvature, in the vicinity of $8.6 \mathrm{~mm}$, and may not exactly fit the shape of the eyes they are aimed to adhere on. Such mismatch can hinder the adhesion of the lens or induce stresses in the eye, leading to undesired discomfort or abrasion issues ${ }^{9}$. How does a contact lens accommodate a possible mismatch in curvature?

Inspired by the practical issue of contact lenses, we designed a model experiment where a thin and shallow cap of radius of curvature $\rho$ and base of radius $a$ is deposited on a solid sphere of different radius $R$ covered with a thin film of wetting liquid acting both as adhesive and lubricant (Fig. 1a). While caps with a base smaller than a critical value $a_{\max }$ can accommodate the difference in curvature, larger specimens lead to complex adhesion features such as blisters, branched stripes or star-shaped bubbles that are reminiscent of the shapes observed in the liquid blister test ${ }^{10}$ (Fig. 1b). The criterion for full adhesion is also valid when the same elastic cap is deposited on the inner surface of a rigid sphere. However, adhesion patterns display a distinct set of morphologies such as circular, annular or oblong contacts (Fig. 1c). We first determine experimentally and theoretically the maximum size of the elastic cap leading to full contact for a given mismatch in curvature, generalizing the work by Hure et $a{ }^{1}{ }^{1}$. We then describe the different patterns obtained beyond this critical size in a configuration diagram where stretching, bending and adhesion energies are compared.

\section{EXPERIMENTAL METHODS}

Elastic caps are produced following the simple procedure developed by Lee et al. ${ }^{11}$ : equal masses of liquid base and catalyst of polyvinyl-siloxane elastomer (Elite Double from Zhermack) are mixed and poured on a rigid hemisphere. After curing, homogeneous shells of radius $\rho$ ranging from 25 to $200 \mathrm{~mm}$, uniform thickness $160<h<800 \mu \mathrm{m}$, Poisson ratio $\nu \simeq 0.5$ and Young modulus $E=1.00 \pm 0.05 \mathrm{MPa}$ or $750 \pm 20 \mathrm{kPa}$ (corresponding to Shore 32 and 22, respectively) can be readily peeled away. Before peeling, the elastic shell is cut along a circle of radius $a$ that becomes the base of the cap. In order to prevent possible suction effects of the cap to the rigid surface, a small hole (with a typical diameter of $500 \mu \mathrm{m}$ ) is punched at the center of the soft cap to equalize pressure.

Caps adhere on the rigid sphere through a thin layer of ethanol mixed with methylene blue to visualize the contact area. As ethanol of surface tension $\gamma=22.0 \pm$ $0.2 \mathrm{mN} . \mathrm{m}^{-1}$ perfectly wets both the cap and the rigid hemisphere, a layer of alcohol remains deposited on both 
a.

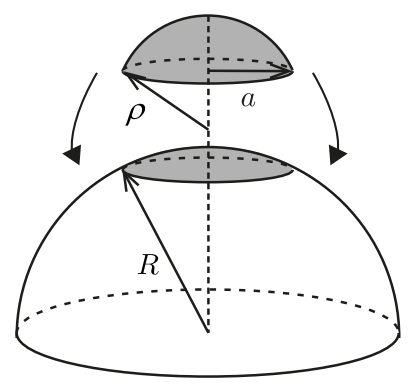

b.
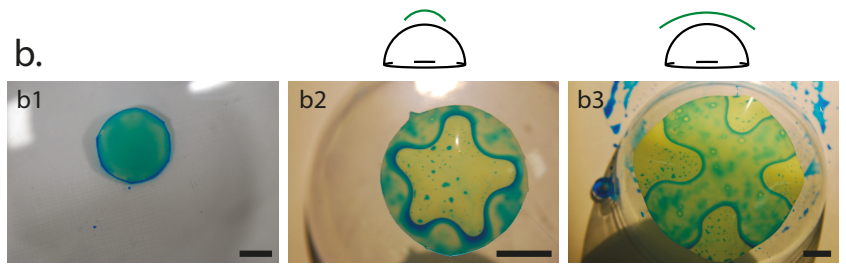

c.
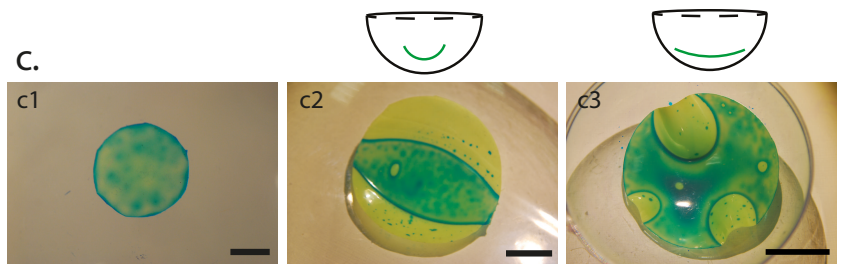

FIG. 1. (a) Sketch of the experiment: an elastic cap of radius of curvature $\rho$ and base of radius $a$ is deposited on a rigid sphere of radius $R$ covered with a layer of ethanol to provide adhesion. Ethanol is dyed with methylene blue to visualize the contact area between the cap and the sphere. Alternatively, the cap can also be deposited against the inner surface of the sphere. (b) Caps deposited on the outer surface of the rigid sphere (top views). For a given curvature mismatch, adhesion is total for caps of narrow base (b1). However, beyond a critical radius $a_{\max }$ of the base, complex adhesion patterns are observed: for instance star shaped bubbles for $\rho<R$ (b2) or branched strips for $\rho>R$ (b3). (c) Caps deposited inside a sphere. While the criterion for full adhesion is preserved (c1), distinct patterns are observed beyond the critical size: circular or oblong patterns for $\rho<R$ (c2) or star-shaped contact for $\rho>R(\mathrm{c} 3)$. Scale bars represent $1 \mathrm{~cm}$.

surfaces upon debonding. Adhesion energy thus corresponds to $2 \gamma$ per unit area.

Based on light absorption, we estimate the thickness of the residual layer of alcohol to be less than $100 \mu \mathrm{m}$ in these contact regions, which is much lower than the millimetric deflection of the cap. Thicker layers are localized in menisci surrounding these regions. The width of the menisci is typically a fraction of millimeter and tends to vanish as alcohol slowly evaporates. In our analysis, we thus neglect the potential effect of the thickness of the layer of liquid in the deformation of the caps.

\section{MAXIMUM SIZE OF THE CAP}

For a given mismatch in curvature, what is the maximum size of the cap leading to total adhesion? Capillary energy promotes adhesion while bending and stretching energies oppose the deformations of the cap ${ }^{12}$. Within the limit of shallow caps $(a \ll R, \rho)$, the adhesion energy is proportional to $\gamma a^{2}$. The bending energy involved in the transformation scales as $E h^{3} a^{2} \Delta C^{2}$, with $\Delta C=1 / R-1 / \rho$ (see derivation in the appendix). Bending is therefore negligible in comparison with capillary adhesion if $\Delta C \ll 1 / L_{e b}$, where $L_{e b}=\left(\frac{E h^{3}}{12\left(1-\nu^{2}\right) \gamma}\right)^{1 / 2}$ is the classical capillary bending length for a plate ${ }^{13}$. The experiments of this section are carried out in the regime $\Delta C<1 / L_{e b}$, whereas this condition will not be always verified in the next section, beyond the critical size.

Owing to Gauss' theorema egregium, a change in the radius of curvature of a spherical cap also involves distortions of the metrics of the surface. The cap thus accumulates elastic energy due to strains along the surface. For spherical caps $(a \ll \rho)$, the relative difference in the projected radius $a$ and the radius measured along the surface scales quadratically with $a / \rho$. Flattening a cap of curvature $1 / \rho$ and base radius $a$ thus induces strains proportional to $(a / \rho)^{2}$ along the surface. Changing the curvature of a cap from $1 / \rho$ to $1 / R$ thus leads to strains of the order of

$$
\epsilon \sim a^{2}\left|\frac{1}{\rho^{2}}-\frac{1}{R^{2}}\right|
$$

The associated elastic energy scales as $E h a^{2} \epsilon^{2}$, while the capillary adhesion energy is of the order of $\gamma a^{2}$. Balancing both terms gives the maximal size $a_{\max }$ of a fully adhering cap:

$$
a_{\max } \sim R\left(\frac{\gamma}{E h}\right)^{1 / 4} \frac{1}{\sqrt{\left|\left(\frac{R}{\rho}\right)^{2}-1\right|}}
$$

The limit $\rho / R \rightarrow+\infty$ corresponds to the relation obtained by Hure et al. ${ }^{1}$ for a plane sheet adhering on a sphere: $a_{\max } \sim R(\gamma / E h)^{1 / 4}$. Conversely, the opposite limit $R / \rho \rightarrow+\infty$ describes the case of an elastic cap deposited against a flat surface and leads to $a_{\max } \sim \rho(\gamma / E h)^{1 / 4}$. Finally, if the cap and the sphere have the same curvature $(\rho=R)$, no elastic cost opposes adhesion, and $a_{\max }$ is infinite. A more complete derivation of $a_{\max }$, based on the minimization of the potential energy is given in the Appendix and leads to a prefactor equal to 4 .

To test Eq.2, we measure the maximum cap size $a_{\max }$ leading to full adhesion in a range of radii $25<R, \rho<$ $100 \mathrm{~mm}$, and stretching parameter $2.8 \times 10^{-5}<\gamma / E h<$ $1.1 \times 10^{-4}$. The critical size is determined with an uncertainty of $1 \mathrm{~mm}$. In Fig. 2, we represent the experimental values of the normalized maximal adhesion size 


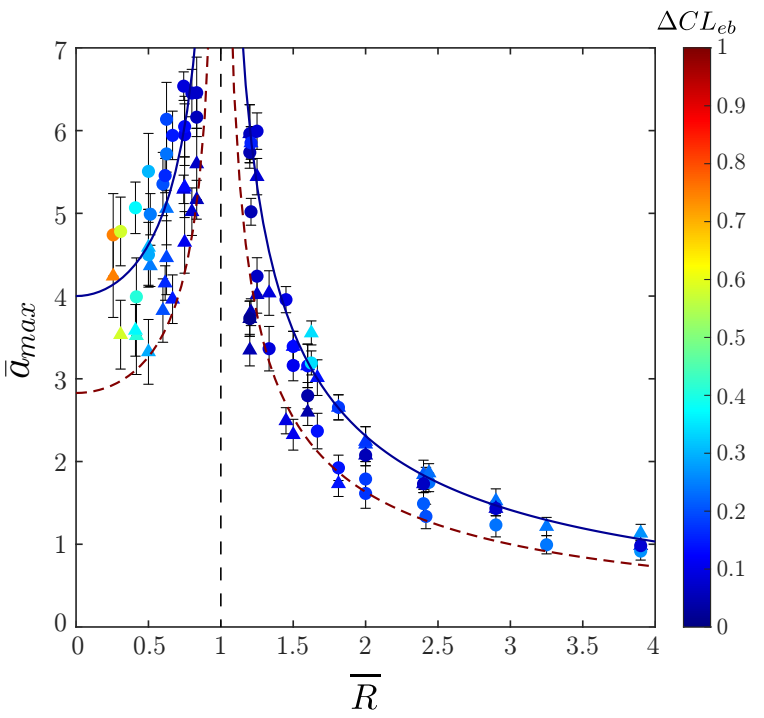

FIG. 2. Normalized maximal adhesion radius $\bar{a}_{\max }$ as a function of $\bar{R}=R / \rho$ as predicted by Eq. 2. Triangles (circles) correspond to caps deposited on the outer (inner) side of the rigid sphere, respectively. The color code represents the value of $\triangle C L_{e b}$ for each data point. The data are well fitted by the asymptotic $\left(\Delta C L_{e b}=0\right)$ theoretical law derived in the Appendix: $\bar{a}_{\max }=4 / \sqrt{\left(\left|1-\bar{R}^{2}\right|\right)}$ (blue solid line). The brown dashed line corresponds to the full law Eq.16 with $\Delta C L_{e b}=1$.

$\bar{a}_{\max }=\frac{a_{\max }}{R}\left(\frac{E h}{\gamma}\right)^{1 / 4}$ as a function of $\bar{R}=R / \rho$. All the data collapse on a single master curve, in good agreement with the theoretical law (Eq. 2, with a prefactor 4 as derived in the Appendix). We attribute the scatter of the data to friction between the cap and the sphere when the liquid layer becomes too thin.

\section{BEYOND THE CRITICAL SIZE}

Caps larger than the critical adhesion radius $a_{\max }$ present complex contact patterns as illustrated in Figs. 1b and 1c. The physical parameters in our experiments are $E, \gamma, \rho, R, a$ and $h$. They are expressed using two independent units, namely lengths and forces. From the Vaschy-Buckingham theorem, the complete description of this problem should require four dimensionless numbers. However, we have identified three main physical ingredients that are competing: adhesion, bending and stretching energies. We thus expect the different configurations to be described by only two relevant dimensionless numbers comparing respectively stretching and bending energies with capillary adhesion. Natural choices are the ratios $a / a_{\max }$ for stretching $v s$ adhesion and $1 /\left(L_{e b} \Delta C\right)$ for bending vs adhesion, respectively. Note that the last parameter can be positive or negative depending on $\Delta C=1 / R-1 / \rho$.

For the same curvature mismatch $\Delta C$, the adhesion patterns differ significantly as the cap is pressed outside or inside the sphere. We thus present the various configurations in two separate diagrams in Figures 3a,b.

\section{A. Contact lens configuration: soft caps outside rigid spheres}

Figure 3a summarizes the adhesion patterns observed when the cap is placed outside the sphere. As described above, caps with a base of radius $a$ smaller than $a_{\max }$ fully adhere to the sphere (region (1) of the diagram). As $a$ increases, delamination occurs and contact is only partial. In the limit of stiff caps $\left(1 /\left|L_{e b} \Delta C\right| \ll 1\right)$, adhesion is too weak to bend the cap, which remains mostly undeformed. In this regime, contact is limited to a small region of the cap, defined by the intersection of the undeformed cap and sphere. If the sphere is more curved than the cap $(\Delta C>0)$, contact occurs only in the vicinity the center of the cap (region (2a)). In the opposite situation $(\Delta C<0)$, only the periphery of the cap touches the sphere. The corresponding annular contact preserves an air bubble at the center (region $(2 \mathrm{~b})$ ). Conversely, caps with lower bending modulus $\left(1 /\left|L_{e b} \Delta C\right| \gg 1\right)$ display contact patterns with more complexity and lower symmetry (patterns $(3 \mathrm{a}, \mathrm{b})$ and $(4 \mathrm{a}, \mathrm{b}))$. If $\Delta C>0$, the adhering region takes the form of elongated strips or branched patterns, as observed when a sphere is wrapped with a thin adhesive sheet ${ }^{1}$. For $\Delta C<0$, we observe the central bubble to evolve to star-like shapes with 3 to 8 arms.

For $a>a_{\max }$, the various domains are defined by the relative magnitudes of adhesion and bending energies. As a tentative rationalization, consider the transition from patterns (2a) to (3a). In pattern (3a), the contact area scales as $a a_{\max }$ and the adhesion energy is of order $\gamma a a_{\max }$. As the cap is overcurved by an amount $\Delta C$ over its entire area, the bending energy scales as $E h^{3} a^{2} \Delta C^{2}$. We thus expect the transition between both regimes to occur for $a / a_{\max } \sim 1 /\left(L_{e b} \Delta C\right)^{2}$. Following the same argument, the transitions between various adhesion patterns would occur for different values of the ratio of the adhesion and bending energies, i.e. for $a / a_{\max }=c_{i-j} /\left(L_{e b} \Delta C\right)^{2}$, where $c_{i-j}$ depends on the boundary between adhesion patterns $i$ and $j$. The boundary between adhesion on a disk (pattern (2a)) and along a strip (pattern (3a)) is well described by $c_{2 a-3 a}=5 \pm 1$. For the transition from strip to branched patterns, we find experimentally $c_{3 a-4 a}=0.8 \pm 0.2$. In the region $\Delta C<0$, the transitions appear more continuous, and different patterns are observed in the same region of the parameter space. A more complete classification of the family of patterns may require the dimensionless numbers that we have disregarded. While the selection of the various patterns most likely relies on the competition between adhesion, bending and stretching energies, the expressions for these energies may be different from what 
we propose due to the complex shapes of the patterns.

\section{B. Covering cavities: soft caps inside rigid spheres}

The situation where the soft cap is deposited inside the spherical cavity is described by the same dimensionless parameters (Fig. 3b). We limit our study to $a<R$ to avoid strong geometrical confinement of the cap in the hemisphere. Similarly to the previous case, buckled patterns appear when $a$ exceeds $a_{\max }$. In this regime, stiff caps $\left(1 /\left|L_{e b} \Delta C\right| \ll 1\right)$ tend to maintain their shapes, leading to very partial adhesion with the sphere: small circular contact zone for caps more curved than the sphere $(\Delta C<0)$ and contact along the periphery for $\Delta C>0$. More flexible caps display complex adhesion patterns: elongated contacts (region (III.b) for $\Delta C<0$ or multiple delaminated peripheral areas (region IV.a) for $\Delta C>0$. The boundary between point-like adhesion and strip adhesion can be described as in the case of adhesion outside a sphere: $a / a_{\max }=c_{I I . b-I I I . b} /\left(L_{e b} \Delta C\right)^{2}$, with $c_{I I . b-I I I . b}=0.3 \pm 0.2$. For $\Delta C>0$, the boundaries cannot be described by a law of the form $a / a_{\max } \sim$ $1 /\left(L_{e b} \Delta C\right)^{2}$. In contrast with adhesion outside a sphere, large caps inside a cavity are geometrically confined, which induces additional contacts. Further theoretical and experimental studies are needed to elucidate this complex behaviour.

\section{CONCLUSION}

We studied the adhesion of an elastic cap on a rigid hemisphere of different radius. The mismatch in Gaussian curvatures prevents a complete adhesion, unless the cap is smaller than a critical size. We derived this maximum size analytically by balancing stretching and capillary energies. The theoretical law we obtained describes adequately the experimental data, as shown by the collapse on a single master curve. We described the different delamination patterns observed in a configuration diagram based on two dimensionless numbers $a / a_{\max }$ and $1 /\left(L_{e b} \Delta C\right)$. We derived some simple scaling arguments to account for the boundaries between the different patterns. Nevertheless, the details of the shapes and boundaries between different adhesion patterns in the parameter space still remain a challenge to address theoretically and numerically. Unexplored regimes may arise in the limit of very thin inextensible sheets where the mismatch in Gaussian curvature is accommodated by crumpling ${ }^{14-16}$ or "wrinklogami" ${ }^{17}$. This "contact lens" problem could finally be extended to surfaces of arbitrary Gaussian curvature. The interplay of geometry and adhesion still contains mysteries to unravel.

\section{Conflicts of interest}

There are no conflicts of interest to declare.

\section{Acknowledgements}

This work was partially funded by the Interuniversity Attraction Poles Programme (IAP 7/38 MicroMAST) initiated by the Belgian Science Policy Office and the French ANR SMArT, and Grant-in-Aid for JSPS Fellows (Grant number 15J10394).

\section{APPENDIX}

We derive the energy involved in the adhesion of an elastic shell on a sphere as presented by Majidi et al. for an elastic sheet in contact with a rigid sphere ${ }^{12}$. We first determine the elastic energy corresponding to the deformation of a cap of radius of curvature $\rho$, base radius $a$, thickness $h$ laid on a hemisphere of radius $R$ (as represented in figure 1). We first assume $\rho>R$. In the limit where $a$ is small compared to $R$ and $\rho$, spherical profiles of the sphere and cap are approximated by parabolic profiles $w(r) \simeq-r^{2} / 2 R$ and $\xi(r) \simeq-r^{2} / 2 \rho$.

Radial and azimuthal strains across the thickness of the shell (direction $z$ ) are related to the radial displacement $u$ and to both profiles $w(r)$ and $\xi(r)$ :

$$
\begin{aligned}
\epsilon_{r r}(r, z) & =u^{\prime}+\frac{1}{2}\left(w^{\prime 2}-\xi^{\prime 2}\right)+z \Delta C \\
& =u^{\prime}+\frac{1}{2} r^{2} \Delta \mathcal{K}+z \Delta C \\
\epsilon_{\theta \theta}(r, z) & =\frac{u}{r}+z \Delta C
\end{aligned}
$$

where .' is the derivative with respect to $r, \Delta \mathcal{K}=\frac{1}{R^{2}}-\frac{1}{\rho^{2}}$ is the mismatch in Gaussian curvature and $\Delta C=\frac{1}{R}-\frac{1}{\rho}$ is the difference in mean curvature.

In the absence of shear, the local mechanical equilibrium in the plane of the shell is given by $\frac{d \sigma_{r r}}{d r}=\frac{\sigma_{\theta \theta}-\sigma_{r r}}{r}$. Radial and azimuthal stresses $\sigma_{r r}$ and $\sigma_{\theta \theta}$ are related to strains through Hooke's law ${ }^{18}$, which in the present configuration writes (in the limit of thin shells, $\sigma_{z z} \sim$ $\left.(h / R) \sigma_{r r} \ll \sigma_{r r}, \sigma_{\theta \theta}\right)$ :

$$
\begin{gathered}
\sigma_{r r}=\frac{E}{1+\nu}\left(\epsilon_{r r}+\frac{\nu}{1-\nu}\left(\epsilon_{r r}+\epsilon_{\theta \theta}\right)\right) \\
\sigma_{\theta \theta}=\frac{E}{1+\nu}\left(\epsilon_{\theta \theta}+\frac{\nu}{1-\nu}\left(\epsilon_{r r}+\epsilon_{\theta \theta}\right)\right)
\end{gathered}
$$

The local equilibrium then reduces to the following differential equation:

$$
u^{\prime \prime}+\frac{u^{\prime}}{r}-\frac{u}{r^{2}}=\frac{1}{2}(\nu-3) r \Delta \mathcal{K}
$$


a.
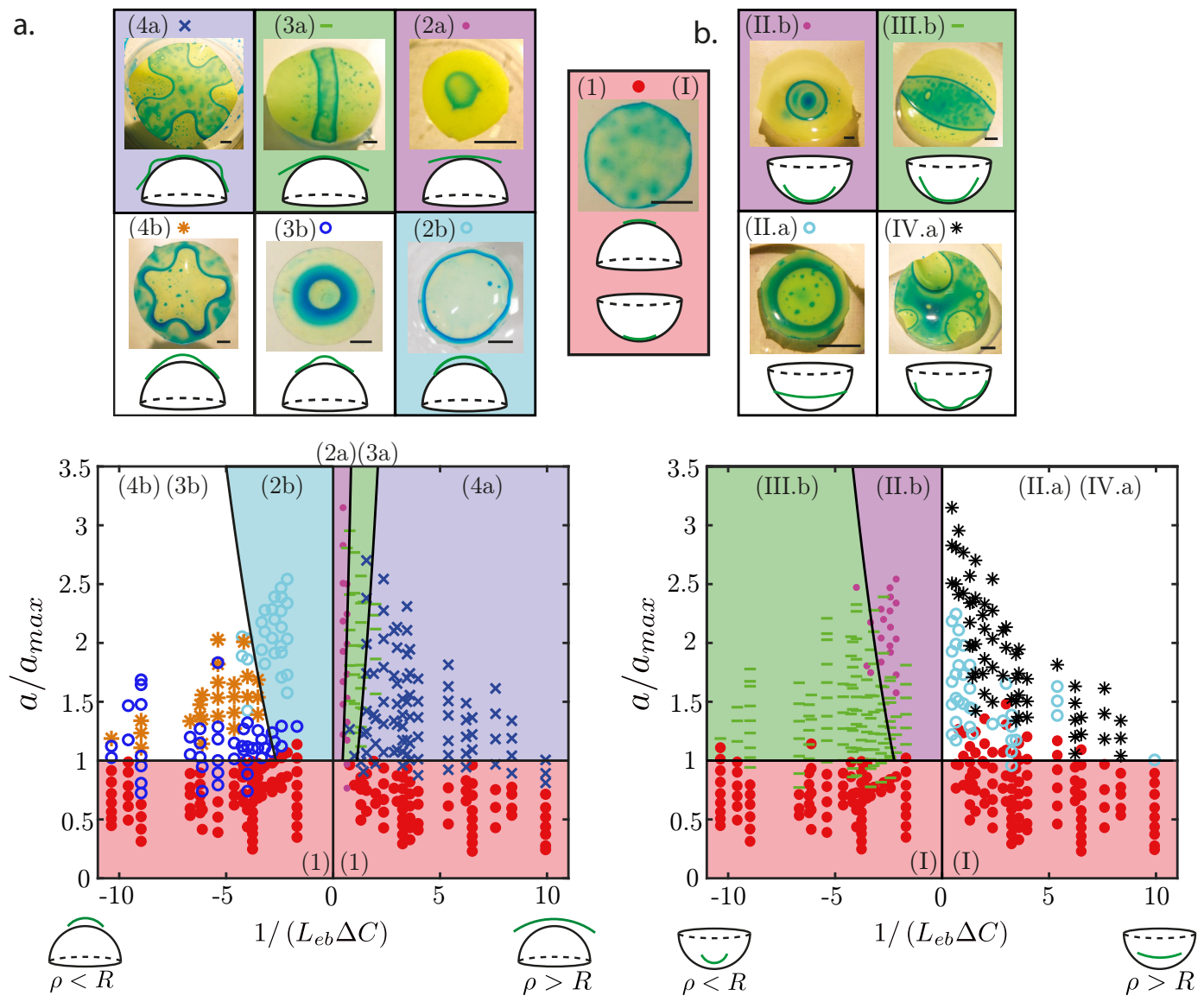

FIG. 3. Diagrams of configuration for the observed adhesion patterns when caps are put on the outside of spheres (a), and when caps are put inside of spheres (b). Different colors and symbols correspond to different patterns. Solid curved lines are scalings for boundaries between different adhesion patterns. Black bars on each picture are $1 \mathrm{~cm}$.

The boundary conditions are $u(0)=0$ by symmetry, and $\sigma_{r r}(a)=0$, which expresses that the edge of the cap is stress-free (we neglect tension created by surface tension). The homogeneous equation is the classical Lamé equation ${ }^{18,19}$, whose solutions are of the form $u(r)=\alpha r+\beta / r$. The $1 / r$ term diverges in 0 and is therefore not present. Moreover, a particular solution of Eq. 7 is $u(r)=\frac{1}{16}(\nu-3) r^{3} \Delta \mathcal{K}$ The solution of Eq. 7 then writes:

$$
u(r)=\frac{1}{16}\left[(1-\nu) r a^{2}+(\nu-3) r^{3}\right] \Delta \mathcal{K}
$$

Using Eqs. 3 and 4, we derive the radial and azimuthal strains:

$$
\begin{aligned}
& \epsilon_{r r}(r, z)=\frac{1}{16}\left[(1-\nu) a^{2}+(3 \nu-1) r^{2}\right] \Delta \mathcal{K}+z \Delta C \\
& \epsilon_{\theta \theta}(r, z)=\frac{1}{16}\left[(1-\nu) a^{2}+(\nu-3) r^{2}\right] \Delta \mathcal{K}+z \Delta C
\end{aligned}
$$

We can now express the elastic energy:

$$
\mathcal{E}_{e l}=\int_{0}^{a} \int_{-h / 2}^{h / 2} 2 \pi r \frac{1}{2} \sigma_{i j} \epsilon_{i j} \mathrm{~d} r \mathrm{~d} z
$$

$\mathcal{E}_{e l}=\frac{E}{2\left(1-\nu^{2}\right)} \int_{0}^{a} \int_{-h / 2}^{h / 2} 2 \pi r\left(\epsilon_{r r}^{2}+2 \nu \epsilon_{r r} \epsilon_{\theta \theta}+\epsilon_{\theta \theta}^{2}\right) \mathrm{d} r \mathrm{~d} z$

The elastic energy may thus be decomposed as the sum of stretching $\mathcal{E}_{s t}$ and bending $\mathcal{E}_{b}$ contributions:

$$
\begin{gathered}
\mathcal{E}_{s t}=\frac{\pi}{384} E h a^{6} \Delta \mathcal{K}^{2} \\
\mathcal{E}_{b}=\frac{E h^{3}}{12(1-\nu)} \Delta C^{2} \pi a^{2}
\end{gathered}
$$

(note that due to the crossed term $\epsilon_{r r} \epsilon_{\theta \theta}$, the dependence of $\mathcal{E}_{b}$ with $\nu$ is not $1 /\left(1-\nu^{2}\right)$ as in the bending of a plate 
along a single direction). The total potential energy $\mathcal{E}_{t o t}$ is the sum of the elastic and the adhesion energies:

$$
\mathcal{E}_{\text {tot }}=\frac{\pi}{384} E h a^{6} \Delta \mathcal{K}^{2}+\frac{E h^{3}}{12(1-\nu)} \Delta C^{2} \pi a^{2}-2 \gamma \pi a^{2}
$$

We minimize $\mathcal{E}_{\text {tot }}$ with respect to $a$ to find $a_{\max }$ :

$$
a_{\max }=\left(\frac{\gamma}{E h} \frac{1}{\Delta \mathcal{K}^{2}}\right)^{1 / 4}\left[256-128(1+\nu)\left(L_{e b} \Delta C\right)^{2}\right]^{1 / 4}
$$

Assuming $\rho<R$ would lead to the same result. The expression for $a_{\max }$ can finally be written:

$$
a_{\max }=\frac{R\left(\frac{\gamma}{E h}\right)^{1 / 4}}{\sqrt{\left|\frac{R^{2}}{\rho^{2}}-1\right|}}\left[256-128(1+\nu)\left(L_{e b} \Delta C\right)^{2}\right]^{1 / 4}
$$

In our experiments $L_{e b} \Delta C$ is small, we can therefore neglect $128(1+\nu)\left(L_{e b} \Delta C\right)^{2}$ in Eq.16, to find:

$$
a_{\max } \simeq 4 R\left(\frac{\gamma}{E h}\right)^{1 / 4} \frac{1}{\sqrt{\left|\frac{R^{2}}{\rho^{2}}-1\right|}}
$$

and recover the scaling law derived in the main text.

\section{BIBLIOGRAPHY}

${ }^{1}$ Hure J, Roman B, Bico J. Wrapping an Adhesive Sphere with an Elastic Sheet. Physical Review Letters. 2011 apr;106(17):174301. Available from: http://link.aps.org/doi/ 10.1103/PhysRevLett.106.174301.

${ }^{2}$ Hure J, Audoly B. Journal of the Mechanics and Physics of Solids Capillary buckling of a thin film adhering to a sphere. Journal of the Mechanics and Physics of Solids. 2013;61(2):450471. Available from: http://dx.doi.org/10.1016/j.jmps. 2012 . 09.016.

${ }^{3}$ Lobkovsky A, Gentges S, Li H, Morse D, Witten TA. Scaling Properties of Stretching Ridges in a Crumpled Elastic Sheet. Science. 1995 dec;270(5241):1482-1485. Available from: http://www.sciencemag.org/cgi/doi/10.1126/science. 270.5241 .1482 .

${ }^{4}$ Struik DJ. Lectures on Classical Differential Geometry. 2nd ed. Dover; 1988.

${ }^{5} \mathrm{Yu}$ TX, Stronge WJ. Wrinkling of a circular elastic plate stamped by a spherical punch. International Journal of Solids and Structures. 1985;21(10):995-1003. Available from: http: //linkinghub.elsevier.com/retrieve/pii/0020768385900514.
${ }^{6}$ Stronge WJ, Sutcliffe MPF, Yu TX. Wrinkling of elastoplastic circular plates during stamping. Experimental Mechanics. 1986 dec;26(4):345-353. Available from: http://link.springer.com/ 10.1007/BF02320150.

${ }^{7}$ Hure J, Roman B, Bico J. Stamping and wrinkling of elastic plates. Physical Review Letters. 2012 aug;109(5):054302. Available from: https://link.aps.org/doi/10.1103/PhysRevLett. 109.054302 .

${ }^{8}$ King H, Schroll RD, Davidovitch B, Menon N. Elastic sheet on a liquid drop reveals wrinkling and crumpling as distinct symmetry-breaking instabilities. Proceedings of the National Academy of Sciences. 2012 jun;109(25):9716-9720. Available from: http://www.pnas.org/cgi/doi/10.1073/pnas. 1201201109.

${ }^{9}$ Funkenbusch GT, Benson RC. The Conformity of a Soft Contact Lens on the Eye. Journal of Biomechanical Engineering. 1996 aug;118(3):341. Available from: http://biomechanical. asmedigitalcollection.asme.org/ article.aspx?doi=10.1115/1.2796016.

${ }^{10}$ Chopin J, Vella D, Boudaoud A. The liquid blister test. Proceedings of the Royal Society A: Mathematical, Physical and Engineering Sciences. 2008 nov;464(2099):2887-2906. Available from: http://rspa.royalsocietypublishing.org/cgi/doi/10. 1098/rspa.2008.0095.

${ }^{11}$ Lee A, Brun PT, Marthelot J, Balestra G, Gallaire F, Reis PM. Fabrication of slender elastic shells by the coating of curved surfaces. Nature Communications. 2016 apr;7:11155. Available from: http://dx.doi.org/10.1038/ncomms11155http: //www nature. com/doif inder/10.1038/ncomms 11155 .

${ }^{12}$ Majidi C, Fearing RS. Adhesion of an elastic plate to a sphere. Proceedings of the Royal Society A: Mathematical, Physical and Engineering Sciences. 2008 may;464(2093):1309-1317. Available from: http://rspa.royalsocietypublishing.org/cgi/doi/10. 1098/rspa.2007.0341.

${ }^{13}$ Bico J, Reyssat E, Roman B. Elastocapillarity: When Surface Tension Deforms Elastic Solids. Annual Review of Fluid Mechanics. 2018;50(1):629-659.

${ }^{14}$ Paulsen JD, Hohlfeld E, King H, Huang J, Qiu Z, Russell TP, et al. Curvature-induced stiffness and the spatial variation of wavelength in wrinkled sheets. Proceedings of the National Academy of Sciences. 2016;113(5):1144-1149. Available from: https://www . pnas.org/content/113/5/1144.

${ }^{15}$ Paulsen JD. Wrapping Liquids, Solids, and Gases in Thin Sheets. Annual Review of Condensed Matter Physics. 2019;10(1):431450.

${ }^{16}$ Paulsen JD, Démery V, Santangelo CD, Russell TP, Davidovitch B, Menon N. Optimal wrapping of liquid droplets with ultrathin sheets. Nature Materials. 2015 08;14:1206. Available from: https://doi.org/10.1038/nmat4397.

${ }^{17}$ Hohlfeld E, Davidovitch B. Sheet on a deformable sphere: Wrinkle patterns suppress curvature-induced delamination. Phys Rev E. 2015 Jan;91:012407. Available from: https://link.aps.org/ doi/10.1103/PhysRevE.91.012407.

${ }^{18}$ Timoshenko S, Woinowsky-Krieger S. Theory of plates and shells. 2nd ed. McGraw-Hill; 1959.

${ }^{19}$ Audoly B, Pomeau Y. Elasticity and Geometry: From hair curls to the non-linear response of shells. OUP Oxford; 2010. 